\title{
Glutamate and substance $P$ coexist in primary afferent terminals in the superficial laminae of spinal cord
}

\author{
(amino acids/peptides/pain/somatosensation/neurotransmitters)
}

\section{Silvia De Biasi ANd Aldo Rustioni}

Departments of Cell Biology and Anatomy and of Physiology, University of North Carolina, Chapel Hill, NC 27599

Communicated by Irving T. Diamond, July 18, 1988

\begin{abstract}
By light microscopic immunocytochemistry it has been previously shown that $\approx 70 \%$ of the neurons in rat dorsal root ganglia are labeled with an antiserum for glutamate conjugated to hemocyanin; the smaller among these neurons are also positive for substance $P$. By using a postembedding ImmunoGold method and electron microscopy, it is shown here that synaptic terminals in the superficial laminae of the spinal cord of rats selectively stain for the same glutamate antiserum. Immunolabeling is in small dome-shaped and in large scalloped synaptic terminals. Scalloped terminals are of two types. One type consists of dark terminals with many agranular vesicles of different size and a few large granular vesicles; these are probably endings of unmyelinated and small myelinated primary afferent fibers. The other type consists of light terminals with small agranular vesicles homogeneous in size with neurofilaments and many mitochondria; these are probably endings of larger myelinated primary afferent fibers. By means of double-labeling electron microscopic immunocytochemistry with colloidal gold particles of two different sizes, it is also shown here that substance $P$ is present in only the dark type of glutamate-labeled scalloped terminals. The primary afferent origin of the terminals labeled by the antisera for glutamate and for substance $P$ is demonstrated by a triple-labeling strategy: immunocytochemistry for both antisera on sections from rats in which dorsal rhizotomy or dorsal root ganglion injection of horseradish peroxidase conjugated to wheat germ agglutinin was performed. It is proposed that glutamate is the neurotransmitter in primary afferents mediating input from different peripheral receptor classes, including nociceptors. Effects of glutamate and substance $P$ on spinal dorsal horn neurons may result from co-release of these two mediators from the same dorsal root afferent terminal.
\end{abstract}

Glutamate meets several criteria as neurotransmitter in at least some dorsal root ganglion (DRG) neurons. Dorsal roots contain more glutamate than ventral roots $(1,2)$; reversal potential of iontophoretically applied glutamate and synaptically evoked responses are similar in spinal neurons $(3,4)$; and glutamate is released upon dorsal root stimulation in spinal cord slices $(5,6)$. Furthermore, D-aspartate, a metabolically inert analogue taken up by the same high-affinity mechanisms as glutamate, selectively labels a fraction of DRG neurons $(7,8)$, and its uptake and release is decreased in the spinal cord of guinea pigs after dorsal rhizotomy (9). Substance $P$ (SP) is also probably associated with the mediation of peripheral input to spinal dorsal horn neurons. This peptide is present in a subpopulation of DRG neurons and in numerous terminals in the superficial laminae of the spinal dorsal horn $(10,11)$; it depolarizes dorsal horn neurons activated by cutaneous stimulation when applied iontophoretically, and it is depleted by chronic deafferentation (12-

The publication costs of this article were defrayed in part by page charge payment. This article must therefore be hereby marked "advertisement" in accordance with 18 U.S.C. $\$ 1734$ solely to indicate this fact.
14). In the DRGs of the rat, up to $70 \%$ of the neurons are labeled with an antiserum for a glutamate-hemocyanin conjugate, and most of the SP-positive DRG neurons are also immunopositive for the conjugate (15).

The presence of high levels of glutamate in DRG perikarya is suggestive of glutamergic transmission in primary afferent fibers; however, more direct evidence of a neurotransmitter role for this amino acid in these afferents would require its presence in primary afferent endings in the spinal dorsal horn. The present electron microscopic immunocytochemical study was undertaken for this purpose. A postembedding colloidal gold approach was chosen to verify, also, whether both glutamate and SP are present in the same dorsal root terminal, as might be expected from their colocalization in DRG neurons (15).

\section{MATERIALS AND METHODS}

Eight male Sprague-Dawley rats $(250 \mathrm{~g})$ anesthetized with chloralhydrate were perfused transcardially with a mixture of $4 \%$ paraformaldehyde $/ 0.2 \%$ picric acid $/ 1.0 \%$ glutaraldehyde. Cervical cord segments were dissected and immersed in the same fixative for several hours at $4^{\circ} \mathrm{C}$. Vibratome sections (50 $\mu \mathrm{m}$ thick) of these segments were waferembedded in Epon after immersion in $0.5 \% \mathrm{OsO}_{4}$. Thin sections from samples of the superficial laminae of the dorsal horn were collected on parlodion-coated slot nickel grids and etched for 30 min with a saturated solution of sodium metaperiodate, followed by $1 \%$ sodium borohydride for $5 \mathrm{~min}$ $(16,17)$. Postembedding immunocytochemistry was by a modification of the method described by Varndell and Polak (18). Preparation of glutamate antiserum was similar to that described by Storm-Mathisen et al. (19): the antiserum was raised in rabbits against glutamate conjugated to keyhole limpet hemocyanin with glutaraldehyde and it was characterized by immunocytochemistry and immunoabsorption (20). SP antiserum, kindly supplied by P. Petrusz (University of North Carolina), was raised in rabbits against SP conjugated to keyhole limpet hemocyanin with glutaraldehyde. Characterization of this antiserum is incomplete, but preliminary results allow the exclusion of cross-reactivity with neurokinin A (L. Abdullah and P. Petrusz, personal communication). Single immunostaining was first performed to determine the optimal dilution of each antibody $(1: 15,000$ for glutamate and 1:10,000 for SP). The primary antibodies were revealed by using colloidal gold particles $(10-\mathrm{nm}$ diameter, Janssen Life Sciences Products, Beerse, Belgium; 20-nm diameter, E-Y Laboratories, San Mateo, CA) coated with goat anti-rabbit IgG. Incubation time for both antisera and for goat anti-rabbit IgG gold probes was $1 \mathrm{hr}$ at room temperature. Double-labeling was performed according to the method of Wang and Larsson (21). Since both antisera were raised in rabbits, after the first cycle of immunostaining (SP antiserum

Abbreviations: DRG, dorsal root ganglion; LGV, large granular vesicles; $S P$, substance $P$. 
and 10-nm gold IgG) free anti-IgG binding sites were denatured by hot paraformaldehyde vapors. The grids were then incubated in the glutamate antiserum and in a gold probe of $20 \mathrm{~nm}$. Immunocytochemical controls included omission of the primary antisera, replacement of one or both of the primary antisera by normal rabbit serum or by a buffer solution, incubation with colloidal gold alone, and incubation with primary antisera preabsorbed with an excess of the appropriate antigen. Nonspecific staining was very low in all the control sections. No differences in the straining pattern were observed when the sequence in which the antibodies and the gold probes were applied to the section was varied. No immunostaining was present in sections that had been treated with hot paraformaldehyde vapors after incubation in the primary antiserum to test the efficacy of thermal denaturation.

To verify the origin of immunopositive terminals, primary afferent endings were identified by either anterograde degeneration or transport. In two rats, C5 dorsal root was sectioned extradurally on the left side. After 24 or $36 \mathrm{hr}$, the animals were perfused as described above and Vibratome sections from C5 spinal segments were wafer-embedded in Epon. In five rats, a $2 \%$ solution of wheat germ agglutinin-horseradish peroxidase conjugate was injected in two to four consecutive cervical DRGs (C4-C7). Injection volume ranged from 0.1 to $0.5 \mu \mathrm{g}$ per ganglion and the survival time was between 22 and $42 \mathrm{hr}$. These rats were perfused with $2.5 \%$ glutaraldehyde and $0.5 \%$ paraformaldehyde in phosphate buffer. Fifty-micrometer-thick Vibratome sections were cut from one or two cervical segments (C5 and $\mathrm{C6}$ ) and the horseradish peroxidase reaction product was histochemically visualized with the $p$-phenylenediamine/pyrocatechol mixture (22). Sections were inspected under light microscope for the successful visualization of the anterograde labeling and then waferembedded. Thin sections from the superficial dorsal horn of the two groups of animals were then processed for the immunocytochemical localization of glutamate and SP as described above.

\section{RESULTS}

With the immunocytochemical procedure used here, terminals labeled by the glutamate and SP antiserum are identified by the presence of large and small gold particles, respectively. Terminals labeled by both antisera are identified by the presence of particles of both sizes. In the same thin section, single- and double-labeled terminals are present amidst unlabeled ones. In terminals immunoreactive for glutamate, gold particles are randomly distributed over the axoplasm (Fig. $1 A$ and $B$ ). Glutamate-labeled terminals in lamina I are mainly small dome-shaped and make asymmetric synapses in the plane of the section on a single dendrite. They contain many round, small, clear vesicles and, in many instances, a variable number of large granular vesicles (LGV). Glutamate-positive terminals of the same type are also numerous in the outer portion of lamina II where they were intermingled with larger scalloped terminals of two types. One type has dark axoplasm, densely packed clear vesicles of various size, and few LGV and mitochondria; these terminals are often arranged in rows and make asymmetric contacts in the plane of the section with several different profiles, some also containing vesicles (Fig. 1A). The other type has light axoplasm, uniformly small clear vesicles, and many mitochondria; these terminals may contain neurofilaments and make asymmetric contacts with fewer elements than the dark scalloped terminals (Fig. $1 B$ ). The light scalloped terminals predominate in the ventral part of inner lamina II, while dark scalloped terminals are mainly present more dorsally in the same lamina.
SP labeling is characterized by the presence of gold particles over LGV in numerous dome-shaped and dark scalloped terminals; light scalloped terminals are not SP positive. SP-positive terminals also contain unlabeled LGV and many small clear vesicles; labeled LGV are often located away from the synaptic zone (Fig. $1 C-E$ ). Labeled LGV are also in varicosities without synaptic specializations in the plane of the section and in small myelinated or unmyelinated axonal profiles. While the origin of dome-shaped terminals may be varied, scalloped terminals in lamina II have morphological characteristics of primary afferent terminals forming the central element of typical synaptic glomeruli in this lamina (23). Scalloped terminals may belong to two different functional classes according to whether they have a light or dark axoplasm (see Discussion). In the present material, the light scalloped type is only labeled by glutamate, while the dark scalloped type can be either single-labeled by glutamate or double-labeled-i.e., by glutamate and SP (Fig. $1 C-E$ ). Of 450 boutons of the dark scalloped type in lamina II, 314 (70\%) are immunopositive for glutamate, $68(15 \%)$ are labeled with both glutamate and SP, and none is single-labeled with SP. In all double-labeled terminals, the two antigens are in different cellular compartments (Fig. $1 C-E$ ): as in single-labeled terminals, SP immunoreactivity is mainly associated with LGV, while glutamate is throughout the axoplasm. Since LGV may not be uniformly distributed within one terminal, the percentage of double-labeled terminals might be actually higher than suggested by the examination of samples from isolated sections. However, a bouton single-labeled in one section did not display the second label or double-labeling even when followed by several serial sections. Terminals containing flattened vesicles are never labeled with either antiserum.

The primary afferent origin of immunopositive terminals could be documented in rats with rhizotomy (Fig. $2 A$ and $B$ ) or DRG injections (Fig. $2 C$ ). In these rats, anterograde labeling was present in scalloped terminals and in some dome-shaped terminals but not in other types of endings. Endings readily recognizable as labeled by either degeneration or lectin transport tended to display weaker immunocytochemical labeling than terminals at earlier stages of degeneration or with relatively faint histochemical reaction products. As a result of this, no attempt was made to gather quantitative data on immunocytochemical labeling of identified primary afferent terminals. In general, primary afferent terminals only positive for SP were more easily found in rats subjected to rhizotomy than in rats with DRG injections of tracer. Numerous primary afferent terminals only positive for glutamate were found in both groups of animals. Primary afferent endings labeled for both SP and glutamate, of the dark scalloped type in lamina II, were observed after rhizotomy (Fig. $2 A$ and $B$ ) and after tracer injections (Fig. 2C).

\section{DISCUSSION}

Immunostaining with the glutamate antiserum used here is selective in that $(i)$ not all terminals are labeled, (ii) labeled terminals have morphological features associated with the release of an excitatory transmitter, and (iii) terminals containing flattened vesicles, usually associated with the presence of an inhibitory transmitter, especially $\boldsymbol{\gamma}$-aminobutyric acid, are not labeled. Similar selective staining in superficial laminae of the medullary dorsal horn was previously shown by preembedding immunocytochemistry with the same glutamate antiserum used here (24). The likelihood that this selectivity is related to a neurotransmitter pool rather than a metabolic pool has been discussed in previous publications $(24,25)$. Although the possibility remains open that the immunocytochemical label may correlate with high levels of oligopeptides, or neuroactive substances other than 

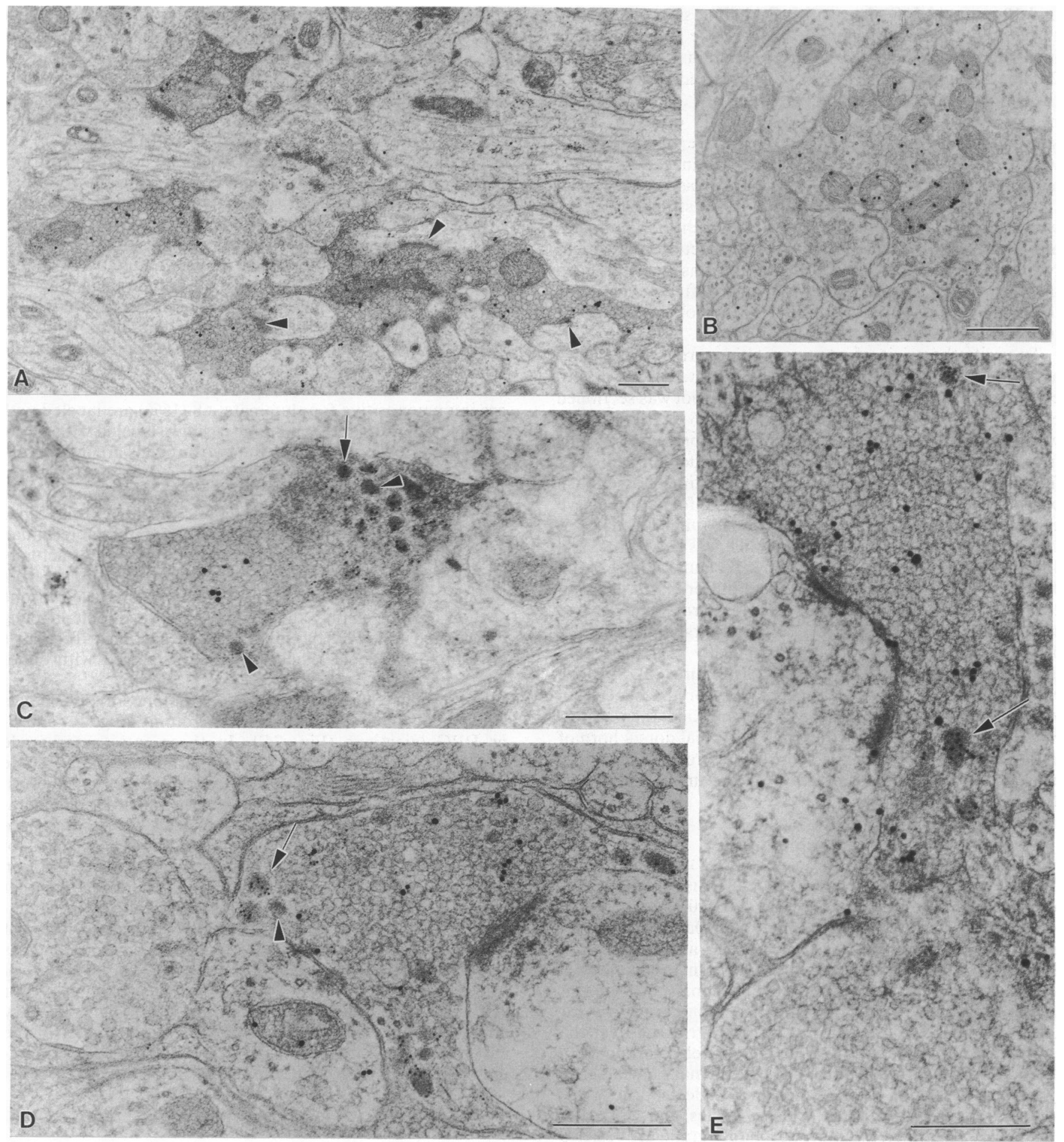

FIG. 1. ( $A$ and $B)$ Scalloped glutamate-labeled (20-nm gold particles) terminals in lamina II of rat dorsal horn. $(A)$ Dark type with densely packed round clear vesicles of varied size and sparse mitochondria; each one of these terminals may make synaptic contacts with several dendrites in the plane of the section (arrowheads). $(B)$ Light type with sparser vesicles uniform in size and with several mitochondria. $(C-E)$ Dark scalloped terminals in lamina II double-labeled by glutamate (large gold particles) and by SP (10-nm gold particles) antisera. Glutamate immunoreactivity is scattered in the axoplasm. SP immunoreactivity is mostly over LGV (arrows), but not all LGV are labeled (arrowheads). $($ Bars $=0.5 \mu \mathrm{m}$.)

glutamate, glutamergic transmission in DRG neurons has been proposed by several lines of evidence summarized in the Introduction. Furthermore, a high concentration of binding sites for glutamate and for $N$-methyl-D-aspartate-type receptors has been reported in superficial laminae of the dorsal horn (26, 27). Therefore, the selective staining of synaptic terminals with the glutamate antiserum is taken as strongly suggestive of a neurotransmitter role for glutamate in the labeled terminals. SP labeling in the superficial laminae of the dorsal horn as reported here is similar to that previously obtained with preembedding immunocytochemistry using different SP antigens $(11,28)$. With regard to cellular localization of SP, the colloidal gold postembedding method allows better assessment of the virtually exclusive association of the peptide with LGV than has been possible in the central nervous system using other immunocytochemical methods. 

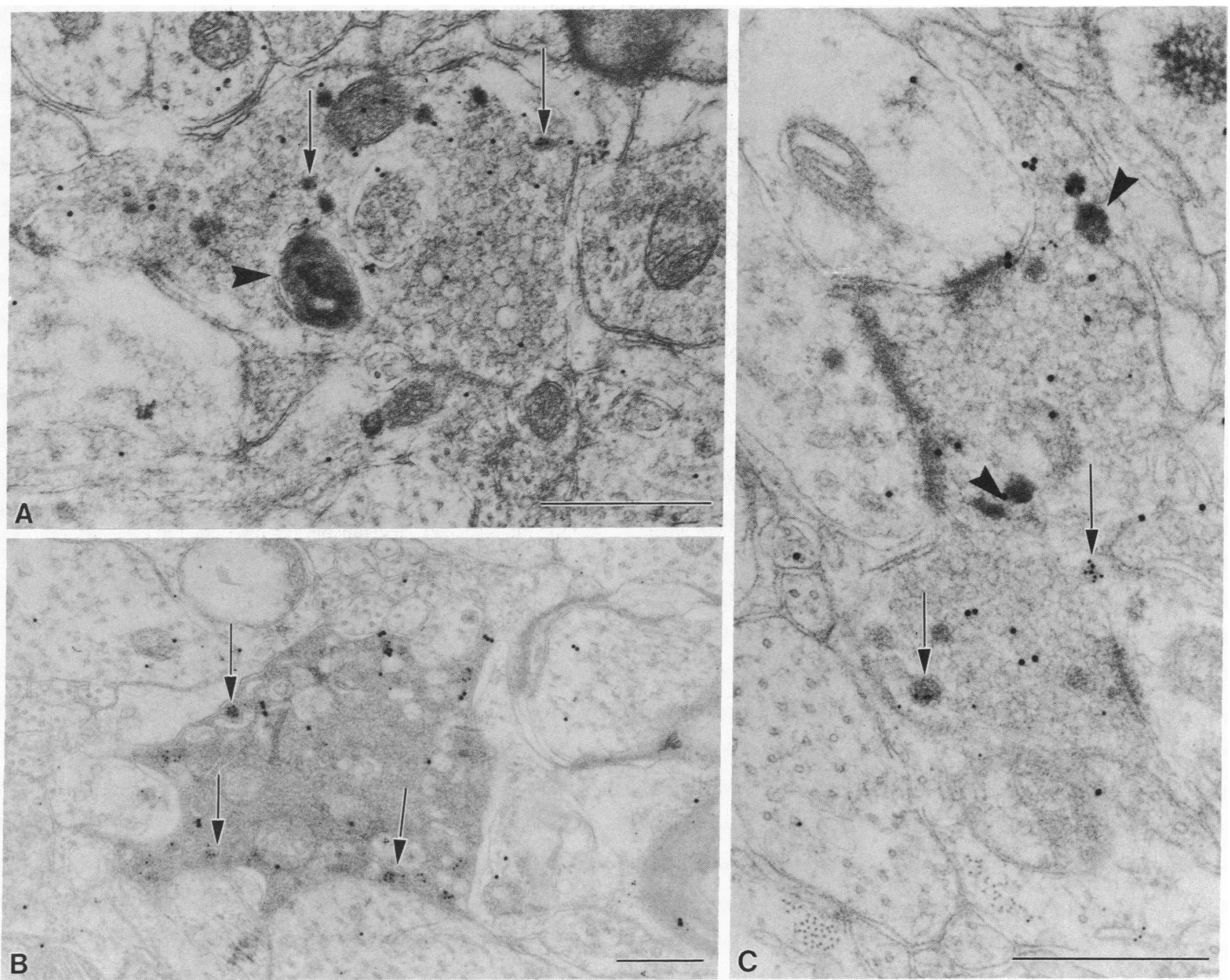

Fio. 2. Primary afferent terminals of the dark scalloped type immunopositive for glutamate (20-nm gold particles) and for SP (10-nm gold particles; arrows). ( $A$ ) Early stage of degeneration characterized by the presence of an osmiophilic body (arrowhead) and clustering of synaptic vesicles. $(B)$ Later stage of degeneration characterized by increased electron density of the axoplasm and shrinkage of the terminal. $(C)$ Electron-dense horseradish peroxidase reaction product (arrow) after injection of wheat germ agglutinin-horseradish peroxidase in the DRG. (Bars $=0.5 \mu \mathrm{m}$.)

The majority of primary afferent terminals in lamina II of the spinal cord, identified here by rhizotomy or anterograde transport, are of the scalloped type: among these terminals the light type is believed to belong to myelinated fibers and the dark type to unmyelinated $(C)$ and small myelinated (AS) fibers $(29,30)$. Both types are glutamate immunopositive, in parallel with the light microscopic observation that both large and small neurons in the DRGs are glutamate immunopositive (15). Primary afferents mediating impulses to the dorsal horn from several different peripheral receptor classes may thus be glutamergic. The present results suggest a role for glutamate in nociception since dark scalloped terminals (from $A \delta$ and $C$ fibers) are glutamate immunopositive; this conclusion is in keeping with the fact that excitation of spinal neurons by $A \delta$ and $C$ fibers is blocked by antagonists of amino acid receptors $(31,32)$. Evidence from iontophoretic release of glutamate in spinal cord slices also suggests that glutamate is the neurotransmitter for nociceptive afferents (33). The coexistence of SP in a fraction of terminals of the same type also argues in favor of glutamergic transmission in nociceptive afferents since this peptide has long been associated with afferents mediating noxious stimuli (34).

Previous electrophysiological studies both in vivo and in vitro have suggested that the synaptic release of SP may function as modulator of the excitatory effects of glutamate $(35,36)$. The present results provide evidence that favors the possibility of a co-release of these two neuromediators from the same primary afferent terminal.

1. Duggan, A. W. \& Johnston, G. A. R. (1970) J. Neurochem. 17, 1205-1208.

2. Salt, T. E. \& Hill, R. G. (1983) Neuroscience 10, 1083-1103.

3. Zieglgänsberger, W. \& Puil, E. A. (1973) Exp. Brain Res. 17, $35-49$.

4. Engberg, I. \& Marshall, K. C. (1979) Neuroscience 4, 15831591.

5. Jessell, T. M., Yoshioka, K. \& Jahr, C. E. (1986) J. Exp. Biol. 124, 239-258.

6. Kawagoe, R., Onodera, K. \& Takeuchi, A. (1986) Biomed. Res. 7, 253-259.

7. Barbaresi, P., Rustioni, A. \& Cuénod, M. (1985) Somatosens. Res. 3, 57-74.

8. Hunt, S. P. (1983) in Chemical Neuroanatomy, ed. Emson, P. C. (Raven, New York), pp. 53-84.

9. Potashner, S. J. \& Tran, P. L. (1984) J. Neurochem. 42, 11351144.

10. Hökfelt, T. K., Kellerth, J. O., Nilsson, G. \& Pernow, B. (1975) Science 190, 889-890.

11. Barber, R. P., Vaughn, J. E., Slemmon, J. R., Salvaterra, 
R. M., Roberts, E. \& Leeman, S. E. (1973) J. Comp. Neurol. 184, 331-351.

12. Henry, J. L. (1976) Brain Res. 114, 439-452.

13. Jessell, T. M., Iversen, L. L. \& Cuello, A. C. (1978) Brain Res. 152, 183-192.

14. Randić, M. \& Miletic, V. (1977) Brain Res. 128, 164-169.

15. Battaglia, G., Rustioni, A., Altschuler, R. A. \& Petrusz, P. (1987) in Fine Afferent Nerve Fibers and Pain, eds. Schmidt, R. F., Schaible, H. G. \& Vahle-Hinz, C. (VCH, Weinheim, F.R.G.), pp. 78-84.

16. Bendayan, M. \& Zollinger, J. (1983) J. Histochem. Cytochem. 31, 101-109.

17. Nilsson, O., Dahlström, A., Geffard, M., Ahlman, H. \& Ericson, L. (1987) J. Histochem. 35, 319-326.

18. Varndell, I. M. \& Polak, J. M. (1984) in Immunolabeling for Electron Microscopy, eds. Polak, J. M. \& Varndell, I. M. (Elsevier, Amsterdam), pp. 155-177.

19. Storm-Mathisen, J., Leknes, A. K., Bore, A. T., Vaaland, J. L., Edminson, P., Haug, F. M. S. \& Ottersen, O. P. (1983) Nature (London) 301, 517-520

20. Hepler, J. R., Toomim, C., McCarthy, K. D., Conti, F. Battaglia, G., Rustioni, A. \& Petrusz, P. (1988) J. Histochem. Cytochem. 36, 13-22.

21. Wang, B. L. \& Larsson, L. I. (1985) J. Histochem. Cytochem. 33, 47-56.

22. Hanker, J. S., Yeats, P. E., Metz, C. B. \& Rustioni, A. (1977) Histochem. J. 99, 789-792.
23. Maxwell, D. J. \& Réthelyi, M. (1987) Trends Neurosci. 10, 117123.

24. Weinberg, R. J., Conti, F., VanEyck, S. L., Petrusz, P. \& Rustioni, A. (1987) in Excitatory Amino Acid Transmission, eds. Hicks, T. P., Lodge, D. \& McLennan, H. (Liss, New York), pp. 173-176.

25. Conti, F., Rustioni, A., Petrusz, P. \& Towle, A. C. (1987) J. Neurosci. 7, 1887-1901.

26. Greenamyre, T. J., Young, A. B. \& Penney, J. B. (1984) J. Neurosci. 4, 2133-2144.

27. Monaghan, D. T. \& Cotman, C. W. (1985) J. Neurosci. 5, 2909-2919.

28. de Lanerolle, N. C. \& La Motte, C. C. (1983) Brain Res. 274, 31-49.

29. Coimbra, A., Ribeiro-da-Silva, A. \& Pignatelli, D. (1984) Anat Embryol. 170, 27.3-287.

30. Knyihar-Csillik, E., Csillik, B. \& Rakic, P. (1982) J. Comp. Neurol. 210, 357-375.

31. Schneider, S. P. \& Perl, E. R. (1988) J. Neurosci. 8, 2062-2073.

32. Schouenborg, J. \& Sjölund, B. H. (1986) Brain Res. 379, 394-398.

33. Schneider, S. P. \& Perl, E. R. (1985) Brain Res. 360, 339-343.

34. Lynn, B. \& Hunt, S. P. (1984) Trends Neurosci. 7, 186-188.

35. Mayer, C. \& Zieglgänsberger, W. (1987) in Fine Afferent Nerve Fibers and Pain, eds. Schmidt, R. F., Schaible, H. G. \& Vahle-Hinz, C. (VCH, Weinheim, F.R.G.), pp. 249-262.

36. Urban, L. \& Randic, M. (1984) Brain Res. 290, 336-341. 